\title{
PREVALÊNCIA DE ATIVIDADE FÍSICA EM MULHERES
}

\section{Prevalence of physical activity in women}

\author{
Prevalencia de la actividad física en mujeres
}

Artigo Original

\section{RESUMO}

Objetivo: Investigar a prevalência de atividade física (AF) em mulheres de FlorianópolisSC. Métodos: Estudo quantitativo e transversal, desenvolvido entre 2012 e 2013, realizado com 400 mulheres entre 20 e 59 anos da região urbana da cidade de Florianópolis. Utilizouse um questionário autoaplicável contendo dados demográficos, peso e altura. Aplicou-se o questionário internacional de atividade física (IPAQ). Para a comparação das variáveis da atividade física, utilizou-se a ANOVA, e para comparação dos níveis (insuficientemente ativo, ativo e muito ativo) de atividade física, o teste do Qui-quadrado. Adotou-se nível de significância de 5\% $(\mathrm{p}<0,05)$. Resultados: Aproximadamente $58-76 \%$ das mulheres foram consideradas suficientemente ativas, sendo a atividade física de intensidade moderada a mais prevalente em todos os grupos etários, destacando-se a faixa etária de 46 a 59 anos (74 $\min / \mathrm{d})$. Do total, $58,7 \%(\mathrm{n}=232)$ das mulheres moram com seus parceiros, $81,8 \%(\mathrm{n}=327)$ possuem ensino superior, $74,5 \%(n=298)$ pertencem à classe econômica $B, 46,8 \%(n=187)$ não possuem filhos e $74 \%(n=296)$ apresentam peso normal. No entanto, essas variáveis parecem não influenciar a prática de AF. Conclusão: Ressalta-se o elevado quantitativo de mulheres suficientemente ativas, sendo a atividade física de intensidade moderada mais prevalente em todos os grupos etários. Nesse sentido, elas atendem às recomendações para a prática de atividade física apontadas pelas agências de saúde.

Descritores: Atividade Motora; Mulheres; Prevalência.

\section{ABSTRACT}

Objective: To investigate the prevalence of physical activity (PA) in women from Florianópolis-SC. Methods: Quantitative cross-sectional study conducted from 2012 to 2013 with 400 women aged 20-59 years in the urban area of the city of Florianopolis. A selfadministered questionnaire containing data on demographics, weight and height was used. The International Physical Activity Questionnaire (IPAQ) was applied. ANOVA was used to compare physical activity variables and the chi-squared test was used to compare the levels of physical activity (insufficiently active, active and very active). Significance level was set at 5\% ( $<<0.05)$. Results: Approximately 58-76\% of the women were considered sufficiently active, with a prevalence of moderate physical activity in all age groups, particularly at age 46-59 years $(74 \mathrm{~min} / \mathrm{d})$. In all, $58.7 \%(n=232)$ of the women live with their partners, $81.8 \%$ $(n=327)$ have a higher education degree, $74.5 \%(n=298)$ belong to socioeconomic class $B$, $46.8 \%(n=187)$ do not have children, and $74 \%(n=296)$ have normal weight. However, such variables do not seem to influence the practice of $P A$. Conclusion: The great number of very active women is highlighted and moderate physical activity is the most prevalent in all age groups. Therefore, they meet the recommendations for physical activity established by health agencies.

Descriptors: Motor Activity; Women; Prevalence

\author{
Vanessa Terezinha Ferrari \\ Jonck $^{(1)}$ \\ Amanda Soares ${ }^{(2)}$ \\ Camila da Cruz Ramos de \\ Araujo $^{(3)}$ \\ Zenite Machado ${ }^{(3)}$ \\ Nycolle Martins Reis ${ }^{(3)}$ \\ Adriana Coutinho de Azevedo \\ Guimarães $^{(3)}$
}

1) Bacharel em Educação Física pela Universidade do Estado de Santa Catarina -

UDESC - Florianópolis (SC) - Brasil

2) Instituto de Ensino Superior da Grande Florianópolis - IES - São José (SC) - Brasil

3) Universidade do Estado de Santa Catarina - UDESC - Florianópolis (SC) Brasil

Recebido em: 26/06/2014

Revisado em: 23/07/2014 Aceito em: 03/10/2014 


\section{RESUMEN}

Objetivo: Investigar la prevalencia de la actividad fisica $(A F)$ en mujeres de Florianópolis-SC. Métodos: Estudio cuantitativo y transversal desarrollado entre 2012 y 2013 con 400 mujeres entre 20 y 59 años de la zona urbana de la ciudad de Florianópolis. Se utilizó un cuestionario autoadministrado con datos demográficos, peso y altura. Se aplicó el cuestionario internacional de actividad física (IPAQ). Se utilizó la ANOVA para la comparación de las variables de la actividad fisica y la prueba de Chi-Cuadrado para la comparación de los niveles (insuficientemente activo, activo y muy activo) de la actividad física. Se adoptó el nivel de significancia del 5\% $(p<0,05)$. Resultados: Aproximadamente el $58-76 \%$ de las mujeres fueron consideradas suficientemente activas con prevalencia para la actividad fisica de intensidad moderada (74 min/d) para todos los grupos etarios, principalmente en la franja de edad de 46 a 59 años. Del total, el 58,7\% $(n=232)$ de las mujeres viven con sus compañeros, el $81,8 \%(n=327)$ tienen educación superior, el 74,5\% $(n=298)$ es de la clase económica B, el 46,8\% $(n=187)$ no tiene hijos y el $74 \%(n=296)$ presentan el peso normal. Sin embargo, estas variables parecen no tener influencia en la práctica de la $A F$. Conclusión: Se resalta el elevado número de mujeres suficientemente activas con prevalencia para la actividad física moderada en todos los grupos etarios. En este sentido, las mujeres atienden a las recomendaciones de prácticas de actividad fisica sugeridas por las agencias de salud.

Descriptores: Actividad Motora; Mujeres; Prevalencia.

\section{INTRODUÇÃO}

Mudanças no estilo de vida, observadas nos últimos anos, proporcionaram um aumento da inatividade física, tornando-se um sério problema em várias cidades do mundo ${ }^{(1,2)}$. Dados do World Health Organization (WHO) ${ }^{(3)}$ revelam que $17 \%$ da população mundial é classificada como inativa fisicamente e $60 \%$ não correspondem ao mínimo de atividade física recomendável. Um estudo realizado na região Sul do Brasil revelou que $41 \%$ dos adultos de ambos os sexos eram inativos fisicamente ${ }^{(4)}$. Outro estudo realizado com mulheres dessa mesma região mostrou que a inatividade física identificada foi de $37 \%{ }^{(5)}$.

Destacam-se no estado de Santa Catarina alguns municípios que investigaram a inatividade física, como Joaçaba, que encontrou uma inatividade física global de $57 \%{ }^{(6)}$, Mafra com 10\% ${ }^{(7)}$ e Florianópolis com 46\% dos indivíduos de 25 a $49 \operatorname{anos}^{(8)}$.

A literatura expõe que indivíduos que praticam atividade física regularmente apresentam um menor risco de desenvolver alguns tipos de doenças, como as cardiovasculares, o diabetes, alguns tipos de câncer, obesidade e aspectos de ansiedade e depressão, entre outras morbidades, podendo apresentar também menores taxas de mortalidade ${ }^{(9,10)}$. Nesse contexto, é recomendado que um adulto realize pelo menos 150 minutos de atividade física por semana, para promover benefícios à sua saúde e ser considerado fisicamente ativo ${ }^{(11-13)}$.

Mesmo apresentando a importância da atividade física, julgou-se pertinente investigar a prevalência de atividade física em mulheres do município de Florianópolis, devido à sua localização, que favorece a atividade física, apresentando uma estrutura que estimula sua prática, além de desenvolver alguns programas gratuitos de atividade física em instituições de ensino superior e do próprio poder público municipal.

\section{MÉTODOS}

Tratou-se de um estudo quantitativo e do tipo transversal, que contou com uma amostra não probabilística por conveniência de mulheres na faixa etária de 20 a 59 anos da zona urbana da cidade de Florianópolis-SC, sendo desenvolvido entre os anos de 2012 e 2013.

Apesar de ser uma amostra não probabilística, realizou-se o cálculo do tamanho da amostra ${ }^{(14)}$, baseado na população de 136.558 mulheres de 20 a 59 anos da cidade de Florianópolis, Santa Catarina, Brasil, de acordo com o último censo do Instituto Brasileiro de Geografia e Estatística $^{(15)}$. O cálculo da amostra apresentou um erro de estimativa de $5 \%$ e intervalo de confiança de $95 \%$, sendo incluídas no estudo 400 mulheres.

A fim de abranger o quantitativo determinado pelo cálculo, a coleta de dados ocorreu via on-line e na presença de um dos pesquisadores in loco. Distribuíram-se 460 questionários (presencialmente e on-line), mas apenas 400 foram devolvidos corretamente preenchidos (287 on-line e 113 presenciais), não havendo diferença em relação ao estrato econômico e idade. Trinta e oito estavam preenchidos corretamente e 22 mulheres recusaram-se a participar do estudo, alegando desinteresse para responder ao questionário.

As participantes foram convidadas a participar do estudo voluntariamente, por meio de instituições empregadoras ou prestadoras de serviços no nível da educação, saúde, desporto, religião e seguros, além de participantes como donas de casa.

Coletaram-se as características demográficas (idade, escolaridade, estado civil, número de filhos, cor da pele e estrato econômico); peso e estatura autorreferidos para cálculo do índice de massa corporal - IMC (peso/estatura ${ }^{2}$ ); e atividade física pelo questionário internacional de atividade física (IPAQ - versão curta).

Para o registro do estrato econômico, utilizou-se o critério padrão de classificação econômica, o qual classifica a população em classes econômicas A, B, C, D e E, por 
meio da conversão da soma de pontos referentes ao grau de instrução da mulher pesquisada e à quantidade de bens de consumo existentes em casa ${ }^{(16)}$.

Para classificação do IMC (estado de peso), utilizou-se o protocolo da Organização Mundial de Saúde ${ }^{(17)}$, ou seja, baixo peso (IMC $<18,5 \mathrm{~kg} / \mathrm{m}^{2}$ ), eutrofia (IMC $18,5-24,9 \mathrm{~kg} /$ $\mathrm{m}^{2}$ ), sobrepeso (IMC $25,0-29,9 \mathrm{~kg} / \mathrm{m} 2$ ), pré-obesidade e obesidade (IMC $>30,0 \mathrm{~kg} / \mathrm{m}^{2}$ ). Para fins estatísticos, foram reagrupadas em variável dicotômica: peso normal (abaixo do peso e peso normal) e acima do peso (sobrepeso, préobesidade e obesidade).

A atividade física foi avaliada por meio do questionário internacional de atividade física (IPAQ - versão curta), validado pelo Centro de Estudos do Laboratório de Aptidão Física de São Caetano do Sul (CELAFISCS), que é o centro coordenador do IPAQ no Brasil ${ }^{(18)}$. Na avaliação da atividade física habitual através do IPAQ, quantificou-se o número de vezes em que um indivíduo realizou ao menos 10 minutos contínuos de caminhada, de atividade física de intensidade moderada e de intensidade vigorosa na última semana, em diversos domínios (laboral, doméstico, lazer, recreativo e desportivo). Considerando-se que à caminhada é geralmente atribuído um nível de exigência de 3,3METs ${ }^{(18)}$, ou seja, um nível superior ao valor de corte para a atividade física moderada (3METs), a atividade física total, que resulta do somatório da caminhada, da atividade moderada e da atividade vigorosa, corresponde a uma atividade de intensidade no mínimo moderada. A atividade física foi classificada em caminhada, atividade física moderada, moderada + vigorosa, vigorosa e total em minutos/dia.

A análise estatística foi realizada por meio do Statistical Package for Social Sciences (SPSS) - versão 16. Fez-se uso da estatística descritiva, mediante o cálculo de média, desvio padrão e percentual. Para a comparação das variáveis da atividade física com cada faixa etária $(20-32$; 33-45; 4659 anos), utilizou-se a ANOVA. A prevalência dos níveis de atividade física (insuficientemente ativo, ativo e muito ativo) foi expressa em percentagem, determinada a partir da razão entre o número de participantes em cada nível de atividade física e o número total de participantes em cada faixa etária. Para comparação dos níveis de atividade física entre as três faixas etárias, utilizou-se o teste do Qui-quadrado. Fez-se uso ainda do método de extração análise do componente principal entre as variáveis: idade, escolaridade, filhos, índice de massa corporal, estrato econômico e estado conjugal junto ao nível de atividade física (insuficientemente ativo, ativo e muito ativo). O nível de significância estatístico adotado nas análises de comparação entre grupos e de associações entre variáveis foi de $5 \%(\mathrm{p}<0,05)$.
Todas as participantes foram informadas dos objetivos e procedimentos do estudo e assinaram o Termo de Consentimento Livre e Esclarecido (TCLE), conforme a Resolução $n^{\circ}$ 466/12 do Conselho Nacional de Saúde. O estudo foi aprovado pelo Comitê de Ética - Pesquisa em Seres Humanos da Universidade do Estado de Santa Catarina em 02/03/12, protocolo $\mathrm{n}^{\circ}$ 214/2011.

\section{RESULTADOS}

A Tabela I apresenta as características da amostra composta por mulheres de 20 a 59 anos, divididas em 3 grupos etários $(1=20$ a $33,2=34$ a $45,3=46$ a 59 anos). Observou-se que $58,7 \%(\mathrm{n}=232)$ das mulheres moram com seu parceiro, com destaque para o grupo $2(75,5 \% ; n=111)$. Do total, $81,8 \%(\mathrm{n}=327)$ possuem ensino superior, $74,5 \%$ $(n=298)$ pertencem a classe econômica $B, 46,8 \%(n=187)$ não possuem filhos e $74 \%(\mathrm{n}=296)$ apresentam peso normal, destacando-se o grupo 1 , com $82,2 \%(n=152)$. Com exceção do ensino superior, todas as variáveis apresentaram associação significativa entre o grupo etário e as variáveis estado conjugal $(\mathrm{p}<0,001)$, número de filhos $(\mathrm{p}<0,001)$, estado de peso $(\mathrm{p}<0,001)$ e classe econômica $(\mathrm{p}=, 032)$.

A Tabela II mostra a prática da atividade física realizada pelas mulheres, nomeadamente: caminhada, atividade física de intensidade moderada, vigorosa e total por estrato etário. Apesar de não haver diferença significativa, a atividade física de intensidade moderada foi a mais praticada em todos os grupos etários, principalmente na faixa etária de 46 a 59 anos, num total de $74 \mathrm{~min} /$ d. Já a caminhada foi considerada menos praticada em questão de minutos/dia quando comparada as demais, sendo ainda menos incidente na faixa etária de 20 a 32 anos com $42 \mathrm{~min} / \mathrm{d}$.

O nível de atividade física foi apresentado na Tabela III, na qual se observa o elevado quantitativo de mulheres suficientemente ativa (ativas + muito ativas), havendo destaque para o grupo 3, com $76 \%(\mathrm{n}=40)$ das mulheres consideradas muito ativas. Entretanto, não houve diferença significativa entre os grupos etários e o nível de atividade física ( $\mathrm{p}=0,431)$.

Na Figura 1, observa-se que apenas duas variáveis se destacaram das demais quanto ao seu peso $(56 \%)$ em relação à atividade física, nomeadamente a idade e a escolaridade, valor considerado baixo, o que significa que apenas a idade e a escolaridade parecem influenciar, mesmo que de maneira fraca, a prática de atividade física.

Verifica-se na Tabela IV que não houve diferença na análise de igualdade de variância. As variáveis nomeadas na tabela parecem não influenciar a prática de atividade física (aumento ou diminuição). 
Tabela I - Caracterização da amostra de acordo com o estrato etário das mulheres participantes do estudo. Florianópolis - SC, $2012-2013$.

\begin{tabular}{|c|c|c|c|c|c|}
\hline Variáveis n(\%) & Total & $\begin{array}{c}\text { Grupo } 1 \\
(20 \text { a } 32 \text { anos })\end{array}$ & $\begin{array}{c}\text { Grupo } 2 \\
\text { (33 a } 45 \text { anos) }\end{array}$ & $\begin{array}{c}\text { Grupo } 3 \\
\text { (46 a } 59 \text { anos) }\end{array}$ & Valor de $p$ \\
\hline \multicolumn{6}{|l|}{ Estado Conjugal (\%) } \\
\hline Morando Junto & $232(58,7)$ & $74(41,1)$ & $111(75,5)$ & $47(69,1)$ & $<0,001$ \\
\hline Morando separado & $163(41,3)$ & $106(58,9)$ & $36(24,5)$ & $21(30,9)$ & \\
\hline \multicolumn{6}{|l|}{ Escolaridade (\%) } \\
\hline Ensino fundamental & $14(3,5)$ & $5(2,7)$ & $4(2,7)$ & $5(7,4)$ & 0,132 \\
\hline Ensino médio & $59(14,7)$ & $28(15,1)$ & $26(17,4)$ & $5(7,4)$ & \\
\hline Ensino superior & $327(81,8)$ & $152(82,2)$ & $117(79,6)$ & $58(85,3)$ & \\
\hline \multicolumn{6}{|l|}{ Classe Econômica(\%) } \\
\hline A & $72(18,0)$ & $28(15,1)$ & $25(17)$ & $19(27,9)$ & 0,032 \\
\hline B & $298(74,5)$ & $137(74,1)$ & $114(77,6)$ & $47(69,1)$ & \\
\hline $\mathrm{C}$ & $30(7,5)$ & $20(10,8)$ & $8(5,4)$ & $2(2,9)$ & \\
\hline \multicolumn{6}{|l|}{ Filhos (\%) } \\
\hline Nenhum & $187(46,8)$ & $143(77,2)$ & $34(23,1)$ & $10(14,7)$ & $<0,001$ \\
\hline 1 a 2 filhos & $183(45,7)$ & $41(22,3)$ & $99(67,3)$ & $33(63,2)$ & \\
\hline 3 a 4 filhos & $30(7,5)$ & $1(0,5)$ & $14(9,5)$ & $15(22,1)$ & \\
\hline \multicolumn{6}{|l|}{ Estado de peso $(\%)$} \\
\hline Peso normal & $296(74,0)$ & $154(83,2)$ & $104(70,7)$ & $38(55,9)$ & $<0,001$ \\
\hline Acima do peso & $104(26,0)$ & $31(16,8)$ & $43(29,3)$ & $30(44,1)$ & \\
\hline
\end{tabular}

Valor de p: nível de significância relativo à comparação da amostra entre os grupos efetuada por meio do Teste Qui quadrado (p<0,05).

Tabela II - Caracterização da atividade física praticada pelas mulheres de acordo com o estrato etário. Florianópolis - SC, $2012-2013$.

\begin{tabular}{lcccc}
\hline Variáveis & $\begin{array}{c}\text { Grupo 1 } \\
\text { (20 a 32 anos) } \\
\text { Média } \pm \text { DP }\end{array}$ & $\begin{array}{c}\text { Grupo 2 } \\
\text { (33 a 45 anos) } \\
\text { Média } \pm \text { DP }\end{array}$ & $\begin{array}{c}\text { Grupo 3 } \\
\text { (46 a 59 anos) } \\
\text { Média } \pm \text { DP }\end{array}$ & Valor de $\boldsymbol{p}$ \\
\hline & & & & \\
Caminhada (min/d) & $42 \pm 47$ & $46 \pm 47$ & $51 \pm 47$ & 0,374 \\
AF moderada (min/d) & $60 \pm 49$ & $69 \pm 57$ & $74 \pm 57$ & 0,133 \\
AF vigorosa (min/d) & $49 \pm 50$ & $47 \pm 56$ & $48 \pm 56$ & 0,966 \\
AF mod + vig (min/d) & $109 \pm 85$ & $115 \pm 89$ & $122 \pm 94$ & 0,537 \\
AF total & $151 \pm 110$ & $162 \pm 111$ & $173 \pm 118$ & 0,343 \\
\hline
\end{tabular}

$\mathrm{AF}$ : atividade física; min/d: minuto/dia; mod + vig: moderada + vigorosa. Valor de $\mathrm{p}$ : ANOVA $(\mathrm{p}<0,05)$.

Tabela III - Nível de atividade física das mulheres de acordo com o estrato etário. Florianópolis - SC, 2012 - 2013.

\begin{tabular}{|c|c|c|c|c|}
\hline Variáveis n(\%) & $\begin{array}{c}\text { Grupo } 1 \\
\text { (20 a } 32 \text { anos) }\end{array}$ & $\begin{array}{c}\text { Grupo } 2 \\
\text { (33 a } 45 \text { anos) }\end{array}$ & $\begin{array}{c}\text { Grupo } 3 \\
\text { (46 a } 59 \text { anos) }\end{array}$ & $\begin{array}{c}\text { Valor de } \\
p \\
\end{array}$ \\
\hline Insuf. Ativo (\%) & $34(16,4)$ & $33(17,4)$ & $15(8,0)$ & \\
\hline Ativo $(\%)$ & $42(24,7)$ & $25(15,2)$ & $13(16,0)$ & 0,431 \\
\hline Muito Ativo (\%) & $109(58,4)$ & $89(67,6)$ & $40(76,0)$ & \\
\hline
\end{tabular}

Insuf. Ativo: insuficientemente ativo; Nível de significância relativo à comparação da prática de atividade física entre os grupos efetuada por meio do Teste Qui quadrado $(\mathrm{p}<0,05)$. 
Tabela IV - Teste de igualdade de médias de grupo relacionado ao nível de atividade física. Florianópolis - SC, 2012 - 2013.

\begin{tabular}{lccccc}
\hline Variáveis & Lambda de Wilks & F & df1 & df2 & Sig. \\
\hline Idade & 0,998 & 0,592 & 1 & 393 & 0,442 \\
Escolaridade & 1,000 & 0,020 & 1 & 393 & 0,888 \\
Estado Conjugal & 0,998 & 0,749 & 1 & 393 & 0,387 \\
Filhos & 1,000 & 0,187 & 1 & 393 & 0,665 \\
IMC & 1,000 & 0,001 & 1 & 393 & 0,973 \\
Estrato econômico & 0,992 & 3,191 & 1 & 393 & 0,075 \\
\hline
\end{tabular}

Análise Discriminante. IMC: Índice de massa corporal

\begin{tabular}{|l|c|c|c|c|c|c|}
\hline \multirow{2}{*}{ Componente } & \multicolumn{3}{|c|}{ Valores próprios iniciais } & \multicolumn{3}{c|}{ Somas de extração de carregamentos ao } \\
& \multicolumn{3}{|c|}{ quadrado } \\
\cline { 2 - 7 } & Total & \% de variação & \% cumulativa & Total & \% de variação & \% cumulativa \\
\hline Idade & 2.063 & 34.386 & 34.386 & 2.063 & 34.386 & 34.386 \\
\hline Escolaridade & 1.327 & 22.108 & 56.495 & 1.327 & 22.108 & 56.495 \\
\hline Estado conjugal & .872 & 14.541 & 71.035 & & & \\
\hline Filhos & .770 & 12.832 & 83.867 & & & \\
\hline IMC & .608 & 10.139 & 94.006 & & & \\
\hline Estrato econômico & .360 & 5.994 & 100.000 & & & \\
\hline
\end{tabular}

IMC: Índice de massa corporal; Método de extração: análise do componente principal. Variação total explicada das variáveis (1=idade; $2=$ escolaridade; $3=$ estado conjugal; 4= filhos; $5=$ IMC; $6=$ estrato econômico).

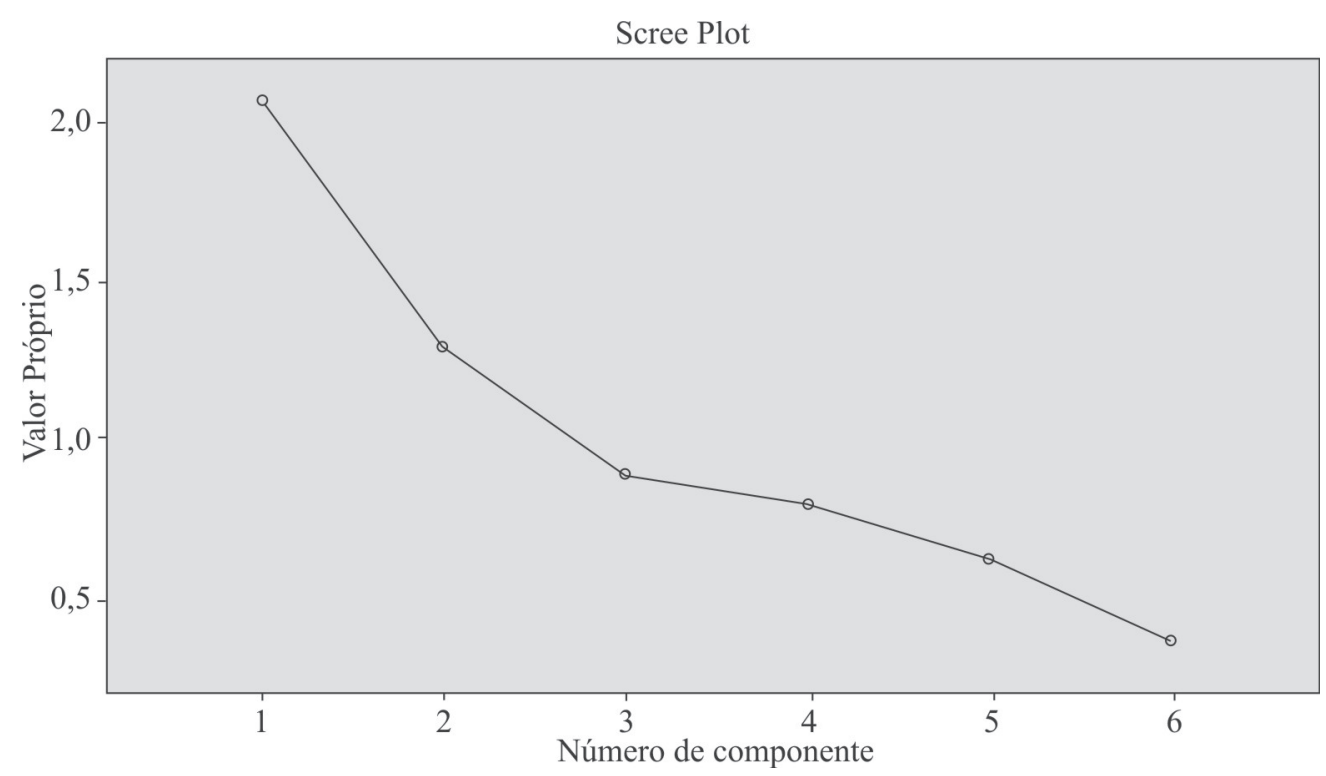

Figura 1 - Variação total explicada das variáveis, idade, escolaridade, filhos, índice de massa corporal, estrato econômico e estado conjugal.

\section{DISCUSSÃO}

A prática de atividade física tem sido relacionada como um dos principais fatores para se manter saudável ${ }^{(19)}$, pois ela pode combater ou prevenir o aparecimento de algumas doenças ${ }^{(20-23)}$. Dessa forma, sua ausência é vista como um fator de risco para a saúde da população ${ }^{(24)}$, sendo responsável por $6 \%$ da mortalidade global ${ }^{(13)}$

Neste estudo, constatou-se que aproximadamente $58-76 \%$ das mulheres são suficientemente ativas. Um levantamento com o mesmo instrumento (IPAQ) foi 
realizado nas cidades de São Caetano do Sul e Pelotas, no qual $60 \%$ e $71 \%$, respectivamente, foram classificadas como suficientemente ativas ${ }^{(25)}$. Corroborando com o que foi observado, outro estudo no Brasil verificou a prática de atividade física em 27 cidades, no qual se percebeu um aumento da prática de atividade física no lazer, quando comparados os dados de 2006 e 2008, respectivamente de $14,9 \%$ para $16,4 \%{ }^{(26)}$. Cabe ressaltar que, nos últimos cinco anos, a prática de atividade física aumentou cerca de 12,6\% para indivíduos brasileiros; esse aumento está atrelado principalmente à procura pela prevenção e promoção da saúde ${ }^{(27)}$.

Segundo as instruções da Global Recommendations on Physical Activity for Heal, indivíduos adultos devem acumular pelo menos 30 minutos de atividade física moderada, cinco vezes por semana, ou 20 minutos de atividade vigorosa três vezes por semana, ou 150 minutos semanais nas atividades de lazer, no deslocamento, no trabalho ou no ambiente doméstico ${ }^{(13)}$.

O fato de Florianópolis apresentar dados sobre a prevalência de níveis de atividade física relativamente maiores em relação a outros estudos nacionais devese, possivelmente, ao fato de ser localizada em uma região litorânea, dispondo de ciclovias, praias, parques e reservas naturais. Corroborando com o descrito, análises por região indicam que os indivíduos residentes no litoral são mais ativos (66,5\%) se comparados aos do interior e áreas metropolitanas $(53,4 \%$ e $39,4 \%$, respectivamente), e afirmam ainda que as pessoas fazem uso da caminhada e ou bicicleta como meio de transporte/deslocamento, favorecendo consideravelmente a prática de atividade física ${ }^{(28)}$. Ademais, a caminhada é uma atividade natural, que pode ser realizada em ambientes abertos, propiciando benefícios à saúde ${ }^{(29)}$.

No presente estudo, verificou-se que a atividade física de intensidade moderada foi prevalente em todos os grupos etários, destacando-se na faixa etária de 46 a 59 anos, totalizando $74 \mathrm{~min} / \mathrm{d}$. Esses resultados corroboram com os achados na literatura, segundo os quais a maioria das mulheres praticava atividade física de intensidade moderada $^{(30)}$. Um estudo internacional realizado em 20 países verificou que a maior parte dos indivíduos entrevistados realizava atividades físicas moderadas ${ }^{(31)}$. A prática de atividade física apresenta aspectos positivos na saúde nas mais diversas intensidades (leve, moderada e vigorosa), sendo a moderada e a vigorosa as que obtêm os maiores benefícios ${ }^{(32)}$.

Realizada regularmente em intensidade moderada, a atividade física pode melhorar a saúde e diminuir o risco precoce de morte ${ }^{(33)}$. Fatores como estado civil, número de filhos, escolaridade, nível socioeconômico, composição corporal, estado de saúde e atividade física têm sido indicados como determinantes para um estilo de vida ativo $^{(34)}$.

Os resultados do presente estudo verificaram que a maioria das mulheres é casada e não possui filhos. Em concordância, um estudo observou que mulheres solteiras, em comparação com as casadas e com filhos, possuem maior percepção sobre a importância da prática de exercícios para a saúde ${ }^{(35)}$. Esse fato pode ser observado levando em consideração que essas mulheres buscam por serviços preventivos de saúde, tendendo a receber maiores informações sobre os riscos de acúmulo de gordura na região abdominal, com isso, acabam se direcionando para alguma prática de atividade física ${ }^{(2)}$.

A maioria das participantes deste estudo apresenta nível superior completo. Um estudo transversal realizado com mulheres adultas de São Leopoldo-RS constatou que quanto menor a escolaridade, maior a prevalência de sedentarismo dessas mulheres ${ }^{(36)}$. Atrelado a esse contexto, outro estudo verificou que indivíduos com maiores níveis educacionais têm mais acesso a conhecimento e condições materiais de vida, favorecendo práticas e hábitos saudáveis ${ }^{(4)}$.

A classe econômica B predominou neste estudo. Acredita-se que pessoas dessa classe econômica são poupadas de trabalhos domésticos, por isso, dispõem de maior tempo para realizar atividades físicas ${ }^{(37)}$. Quanto à composição corporal do referido estudo, observou-se que de $55,9 \%$ a $83,2 \%$ das mulheres obtiveram o índice de massa corporal normal. Corroborando com esses achados, um estudo longitudinal mostrou que indivíduos ativos possuem média de IMC dentro da normalidade ${ }^{(38)}$. Um estudo transversal verificou que $72,3 \%$ das participantes apresentaram estado nutricional na faixa de normalidade, sendo ainda classificadas como ativas $63 \%{ }^{(39)}$.

A promoção de estilos de vida mais ativos e a diminuição da inatividade física parecem ser fatores determinantes para a melhoria dos índices de saúde populacional e dos custos relacionados com a gestão dos serviços de saúde ${ }^{(34)}$.

Como na maioria dos estudos sobre atividade física, esta foi avaliada por meio de um questionário, o qual representa uma limitação deste estudo, uma vez que esse método de avaliação está associado com uma estimativa de erro de mais de $35-50 \%{ }^{\left({ }^{(0)}\right)}$. Recomendações para a atividade de melhoria da saúde física são, no entanto, baseadas em estudos epidemiológicos que utilizaram a mesma metodologia, ou seja, com um erro semelhante.

Por fim, chama-se atenção para a necessidade de futuras pesquisas que avaliem os aspectos trabalhados neste estudo, estendendo-os a outras populações e contextos socioculturais, a fim de verificar a generalização desses dados. 


\section{CONCLUSÃO}

Ressalta-se o elevado quantitativo de mulheres suficientemente ativas, sendo a atividade física de intensidade moderada a mais prevalente em todos os grupos etários. Nesse sentido, elas atendem às recomendações para a prática de atividade física apontadas pelas agências de saúde.

\section{REFERÊNCIAS}

1. Trinh OT, Nguyen ND, Dibley MJ, Phongsavan P, Bauman AE. The prevalence and correlates of physical inactivity among adults in Ho Chi Minh City. BMC Public Health. 2008;9(8):204.

2. Silva SPS, Sandre-Pereira G, Salles-Costa R. Fatores sociodemográficos e atividade física de lazer entre homens e mulheres de Duque de Caxias/RJ. Ciênc Saúde Coletiva. 2011;16(11):4491-501.

3. World Health Organization - WHO. The World Health Report 2002: reducing risks, promoting healthy life. Geneva: WHO; 2002.

4. Hallal PC, Victora CG, Wells JC, Lima RC. Physical inactivity: prevalence and associated variables in Brazilian adults. Med Sci Sports Exerc. 2003;35(11):1894-900.

5. Costa PRF, Assis AMO, Silva MCM, Santana MLP, Dias JC, Pinheiro SMC, et al. Mudança nos parâmetros antropométricos: a influência de um programa de intervenção nutricional e exercício físico em mulheres adultas. Cad Saúde Pública. 2009;25(8):1763-73.

6. Baretta E, Baretta M, Peres KG. Nível de atividade física e fatores associados em adultos no Município de Joaçaba, Santa Catarina, Brasil. Cad Saúde Pública. 2007;23(7):1595-602.

7. Flores A. Prevalência da inatividade física e outros fatores de risco relacionados à saúde na população adulta urbana de Mafra - SC [dissertação]. Florianópolis: Universidade Federal de Santa Catarina; 2002.

8. Instituto Nacional de Câncer - INCA. Inquérito domiciliar sobre comportamentos de risco e morbidade referida de doenças e agravos não transmissíveis: Brasil, 15 capitais e Distrito Federal, 2002-2003. Rio de Janeiro: INCA; 2004.

9. Ministério da Saúde (BR). Agita Brasil: guia para agentes multiplicadores. Brasília: Ministério da Saúde; 2002.

10. Shibata A, Oka K, Nakamura Y, Muraoka I. Prevalence and demographic correlates of meeting the physical activity recommendation among Japanese adults. J Phys Act Health. 2009;6(1):24-32.

11. American College of Sports (US). Medicine support federal physical activity guidelines 2011 [acesso em 2013 Set 7]. Disponível em: http://www. acsm.org/about-acsm/media-room/acsm-in-thenews/2011/08/01/acsm-aha-support-federal-physicalactivity-guidelines

12. Haskell WL, Lee I-M, Pate RR, Powell KE, Blair SN, Franklin BA, Macera CA, et al. Physical activity and public health: updated recommendation for adults from the American College of Sports Medicine and the American Heart Association. Med Sci Sports Exerc. 2007;39(8):1423-34.

13. World Health Organization - WHO. Global recommendations on physical activity for health. Geneva: WHO; 2011.

14. Barbetta PA. Estatística aplicada às ciências sociais. 7 ed. rev. Florianópolis: Editora da UFSC; 2008.

15. Instituto Brasileiro de Geografia e Estatística - IBGE. Projeção da população do Brasil/censo 2010 [acesso em 2012 Maio 2]. Disponível em: http//www.ibge.gov. br

16. World Health Organization - WHO. Global Strategy on Diet, Physical Activity and Health. Geneva: WHO; 2004.

17. Associação Brasileira de Empresas de Pesquisa ABEP. Critério de Classificação Econômica Brasil. São Paulo: ABEP; 2010.

18. Pardini R, Matsudo S, Matsudo TAV, Andrade E, Braggion G, Andrade D, et al. Validation of the international physical activity questionnaire (IPAQ): pilot study in brazilian young adults. Med Sci Sports Exerc. 1997;29:S5-S9.

19. Guallar-Castillón P, Bayán-Bravo A, León-Muñoz LM, Balboa-Castillo T, López-García E, GutierrezFisac JL, et al. The association of major patterns of physical activity, sedentary behavior and sleep with health-related quality of life: a cohort study. Prev Med. 2014;67:248-54.

20. Owen N, Healy GN, Matthews CE, Dunstan DW. Too much sitting: the population health science of sedentary behavior. Exerc Sport Sci Rev. 2010;38(3):105-13.

21. Florencio GLD, Gonçalves AKS, Canário ACG, Silva MJP. A. Envelhecimento: uma reflexão sobre actividade física e estresse oxidativo em mulheres. Acta Médica Portuguesa. 2011;24(Supl 4):983-8. 
22. Patten SB, Williams JV, Lavorato DH, Bulloch AG. Recreational physical activity ameliorates some of the negative impact of major depression on health-related quality of life. Front Psychiatry. 2013;4(22):1-5.

23. Guimarães GV, Ciolac EG. Physical activity: practice this idea. Am J Cardiovasc Dis. 2014;4(1):31-3.

24. Amin TT, Al-Hammam AM, Almulhim NA, Al-Hayan MI, Al-Mulhim MM, Al-Mosabeh MJ. Physical activity and cancer prevention: awareness and meeting the recommendations among adult saudis. Asian Pac J Cancer Prev. 2014;15(6):2597-606.

25. Cruciani F, Timóteo A, Matsudo S, Matsudo V. Nível de atividade física de mulheres maiores de 50 anos de idade participantes de um programa de atividade física estruturada. Rev Bras Ciênc Mov. 2009;17(1):1-16.

26. Ministério da Saúde (BR), Vigitel Brasil 2009: vigilância de fatores de risco e proteção para doenças crônicas por inquérito telefônico. Brasília: Ministério da Saúde; 2010.

27. Ministério da Saúde (BR). Aumento na prática de atividades físicas. Brasília: Ministério da Saúde; 2014 [acesso em 2015 Maio 11]. Disponível em: http://www. brasil.gov.br

28. Matsudo SM, Matsudo VR, Araújo T, Andrade D, Andrade E, Oliveira L, et al. Nível de atividade física da população do estado de São Paulo: análise de acordo com o gênero, idade, nível sócio econômico, distribuição geográfica e de conhecimento. Rev Bras Ciênc Mov. 2002;10(4):41-50.

29. Salles-Costa R, Heilborn ML, Werneck GL, Faerstein E, Lopes CS. Gênero e prática de atividade física de lazer. Cad Saúde Pública. 2003;19(Supl 2):S325-S333.

30. Silva RB, Costa-Paiva L, Pinto NAM, Braga AA, Morais SS. Atividade física habitual e risco cardiovascular na pós-menopausa. Rev Assoc Med Bras. 2006;52(4):242-6.

31. Bauman A, Bull F, Chey T, Craig CL, Ainsworth BE, Sallis JF, et al. The International Prevalence Study on Physical Activity: results from 20 different countries. Int J Behav Nutr Phys Act. 2009;6(89):21-32.

32. Mazzeo RS, Cavanagh P, Evans WJ, Fiatarone MA, Hagberg J, McAuley E, et al. Exercício e atividade física para pessoas idosas. Rev Bras Ativ Fís Saúde. 1998;3(1):48-78.
33. Department of Health and Human Services (US). Physical activity guidelines advisory committee report. Washington: Department of Health and Human Services; 2008.

34. Martínez-González MA, Varo JJ, Santos JL, Irala J, Gibney M, Kearney J, et al. Prevalence of physical activity during leisure time in the European Union. Med Sci Sports Exerc. 2001;33(7):1142-6.

35. Branigan KP, O’brien-Cousins S. Older women and beliefs about exercise risks: what has motherhood got to do with it? J Women Aging. 1996;7(4):47-67.

36. Masson CR, Dias-da-Costa JS, Olinto MTA, Meneghel S, Costa CC, Bairros F, et al. Prevalência de sedentarismo nas mulheres adultas da cidade de São Leopoldo, Rio Grande do Sul, Brasil. Cad Saúde Pública. 2005;21(6):1685-94.

37. Florindo AA, Hallal PC, Moura EC, Malta DC. Prática de atividades físicas e fatores associados em adultos, Brasil, 2006. Rev Saúde Pública. 2009;43(Supl 2): $65-73$.

38. Huang Y, Macera CA, Blair SN, Brill PA, Kohl HW 3rd, Kronenfeld JJ. Physical fitness, physical activity, and functional limitation in adults aged 40 and older. Med Sci Sports Exerc. 1998;30(9):1430-5

39. Maciel ES, Sonati JG, Modeneze DM, Vasconcelos JS, Vilarta R. Consumo alimentar, estado nutricional e nível de atividade física em comunidade universitária brasileira. Rev Nutr. 2012;25(6):707-18.

40. Welk G. Physical Activity Assessments for HealthRelated Research. Champaign: Human Kinetics; 2002.

Endereço do primeiro autor:

Vanessa Terezinha Ferrari Jonck

Rua Afonso Pena, 862

Bairro: Estreito

CEP 88070-650 - Florianópolis - SC - Brasil

E-mail: ferrari334@hotmail.com

Endereço para correspondência:

Camila da Cruz Ramos de Araujo

Rua Evaldo Schaeffer, 422 bloco B10 apto 23

Bairro: Jardim Atlântico

CEP 88095350 - Florianópolis - SC - Brasil

E-mail: cami.ramosdearaujo@hotmail.com 